ANNALES

POLONICI MATHEMATICI

$93.2(2008)$

\title{
Note on some variational problem related to statistical solutions of differential equations in Banach spaces
}

\author{
by LECH SŁAWIK (Kraków)
}

Abstract. The properties of statistical solutions for some general differential equations in Banach spaces are investigated.

1. Introduction. Let $X$ be an infinite-dimensional reflexive real Banach space with a basis $\left\{x_{n}\right\}$, endowed with a norm $\|\cdot\|$. Then $\left(X^{*},\|\cdot\|_{*}\right)$ denotes the dual space of $X$. The value of a functional $u^{*} \in X^{*}$ at a point $u$ will be denoted by $\left\langle u^{*}, u\right\rangle$. Let $X_{n}$ be a subspace of $X$ generated by $x_{1}, \ldots, x_{n}$ and let $P_{n}$ denote the projection operator of $X$ onto $X_{n}$ given by the formula

$$
P_{n} x=\sum_{k=1}^{n}\left\langle x_{k}^{*}, x\right\rangle x_{k}, \quad x \in X .
$$

Analogously, for the space $X_{n}^{*}$ generated by $x_{1}^{*}, \ldots, x_{n}^{*}$ the projection operator $Q_{n}$ of $X^{*}$ onto $X_{n}^{*}$ is given by the formula

$$
Q_{n} x^{*}=\sum_{k=1}^{n}\left\langle x^{*}, x_{k}\right\rangle x_{k}^{*}, \quad x^{*} \in X^{*} .
$$

Let $S=[0, T]$ and $F: S \times X \rightarrow X$ be a mapping satisfying the following conditions:

$\left(\mathrm{A}_{1}\right)$ for every $t \in S$ the mapping $F(t, \cdot): X \rightarrow X$ is continuous with respect to the weak topology,

$\left(\mathrm{A}_{2}\right)$ for every $u \in X$ the mapping $F(\cdot, u): X \rightarrow X$ is Bochner measurable,

$\left(\mathrm{A}_{3}\right)$ there exist $b_{1}, b_{2}>0$ such that $\|F(t, u)\| \leq b_{1}+b_{2}\|u\|$ for $u \in X$, $t \in S$.

2000 Mathematics Subject Classification: Primary 34G99; Secondary 49K45, 49K27. Key words and phrases: abstract differential equations, statistical solution. 
We study the differential equation

$$
u^{\prime}(t)=F(t, u(t)) .
$$

In the classical setting, (1.1) is considered with an initial condition

$$
u(0)=a,
$$

but for physical reasons (e.g. turbulance phenomena) it is interesting to consider a statistical approach to (1.1) replacing $a$ by a probability measure $\mu_{0}$.

In [3] we introduced the following definition.

Definition 1.1. Let $\mu_{0}$ be a given probability measure on $\mathcal{B}(X)$ (the $\sigma$ algebra of all Borel subsets of $X$ ) with bounded support. We say that a family $\{\mu(t, \cdot)\}_{t \in[0, T]}$ of probability measures is a statistical solution of (1.1) with initial measure $\mu_{0}$ if the following conditions are satisfied:

(1) For every $u^{*} \in X^{*}$ the function $[0, T] \ni t \mapsto \psi\left(t, u^{*}\right) \in \mathbb{C}$ is absolutely continuous $(\psi(t, \cdot)$ denotes the characteristic functional of the measure $\mu(t, \cdot))$.

(2) For almost all $t \in[0, T]$ and for every $u^{*} \in X^{*}$,

$$
\frac{\partial \psi\left(t, u^{*}\right)}{\partial t}=i \int_{X}\left\langle u^{*}, F(t, u)\right\rangle e^{i\left\langle u^{*}, u\right\rangle} \mu(t, d u) .
$$

(3) For every $u^{*} \in X^{*}$,

$$
\psi\left(0, u^{*}\right)=\int_{X} e^{i\left\langle u^{*}, u\right\rangle} \mu_{0}(d u) .
$$

Existence of a statistical solution was proven in [3]. Unfortunately, such a solution need not be unique. However, analysis of physical events related to this abstract model shows that frequently, apart from an initial condition, additional constraints should be considered. In what follows, we take into account (inspired by [1], [2], [4]) a functional defined on the set of statistical solutions and investigate under what conditions it has a minimum at exactly one point.

2. Variational problem. Let $\mathcal{C}_{b, w}(X)$ denote the vector space of all real functions on $X$ which are weakly continuous and bounded. Let further $\mathcal{M}(X)$ denote the vector space of all bounded real measures on $\mathcal{B}(X)$. As is known, the space $\mathcal{M}(X)$ may be identified with a subspace of $\left[\mathcal{C}_{b, w}(X)\right]^{\prime}$ (the algebraic dual). The space $\mathcal{M}(X)$ separates points in $\mathcal{C}_{b, w}(X)$ so there exists a locally convex topology in $\mathcal{C}_{b, w}(X)$ such that the topological dual $\left[\mathcal{C}_{b, w}(X)\right]^{*}$ is equal to $\mathcal{M}(X)$. Consequently, $\mathcal{M}(X)$ may be regarded as a locally convex topological space with the weak* topology. Let $\mathcal{C}(S, \mathcal{M}(X))$ denote the space of all continuous functions from $S$ into $\mathcal{M}(X)$. This space is considered with the pointwise convergence topology. 
Let $W$ be the set of all families $\mu=\{\mu(t, \cdot)\}_{t \in S}$ of probability measures on $\mathcal{B}(X)$ which are statistical solutions of (1.1) with initial measure $\mu_{0}$ and satisfy the conditions:

(A4) there exists $d>0$ such that for all $\mu \in W, t \in S, R \in \mathbb{R}$,

$$
\int_{E_{R}}\|u\| \mu(t, d u) \leq d \int_{E_{R / d-1}}(1+\|u\|) \mu_{0}(d u)
$$

where $E_{R}=\{u \in X:\|u\|>R\}$,

(A5) the mapping $J: S \ni t \mapsto \int_{X} f(u) \mu(t, d u)$ belongs to $L^{\infty}(S, \mathbb{R})$ for all $f \in \mathcal{C}(X)$ such that $|f(u)| \leq c(1+\|u\|)$ for $u \in X$.

Let $\mathcal{F}: \mathcal{C}(S, \mathcal{M}(X)) \supset W \rightarrow \mathbb{R}$ be a functional which is

(A6) sequentially lower semicontinuous,

(A7) strongly convex.

TheOREm 2.1. Assume that $W \neq \emptyset$. Then there exists exactly one $\bar{\mu} \in W$ such that

$$
\mathcal{F}(\bar{\mu}) \leq \mathcal{F}(\mu), \quad \mu \in W .
$$

Our proof of Theorem 2.1 is based upon the following two lemmas.

Lemma 2.2. Let $\left\{\mu_{n}\right\}_{n \in \mathbb{N}}$ be a sequence of probability measures on $\mathcal{B}(X)$ such that

$$
\int_{X}\|u\| \mu_{n}(d u) \leq M, \quad n \in \mathbb{N} .
$$

Assume that the sequence $\left\{\psi_{n}\right\}$ of characteristic functionals of $\mu_{n}$ is pointwise convergent to a continuous functional $\psi: X^{*} \rightarrow \mathbb{C}$. Then there exists a probability measure $\mu$ on $\mathcal{B}(X)$ such that

$$
\int_{X}\|u\| \mu(d u)<\infty, \quad \psi\left(u^{*}\right)=\int_{X} e^{i\left\langle u^{*}, u\right\rangle} \mu(d u), \quad u^{*} \in X^{*} .
$$

Moreover, for every $f \in \mathcal{C}_{b}(X, \mathbb{R})$ and $k \in \mathbb{N}$,

$$
\lim _{n \rightarrow \infty} \int_{X} f\left(P_{k} u\right) \mu_{n}(d u)=\int_{X} f\left(P_{k} u\right) \mu(d u) .
$$

In the case of a Hilbert space the proof of this lemma can be found in [4]. If $X$ is a reflexive Banach space with basis the proof is similar.

Lemma 2.3. The set $W$ is sequentially compact in $\mathcal{C}(S, \mathcal{M}(X))$.

Proof. Let $\psi_{n}(t, \cdot)$ be the sequence of characteristic functionals of measures $\mu_{n}(t, \cdot), t \in S, n \in \mathbb{N}$. In view of the definition of statistical solution and (A4) we have

$$
\left|\psi_{n}\left(t_{1}, u^{*}\right)-\psi_{n}\left(t_{2}, u^{*}\right)\right| \leq m_{1}\left|t_{1}-t_{2}\right|, \quad u^{*} \in X^{*}, t_{1}, t_{2} \in S,
$$

where $m_{1}>0$ is independent of $n$. 
Directly from the definition of characteristic functional we have

$$
\left|\psi_{n}\left(t, u^{*}\right)-\psi_{n}\left(t, v^{*}\right)\right| \leq m_{2}\left\|u^{*}-v^{*}\right\|, \quad t \in S, u^{*}, v^{*} \in X^{*},
$$

where $m_{2}>0$ is independent of $n$ and $t$.

By inequalities (2.1), (2.2), the family $\psi_{n}, n \in \mathbb{N}$, is equicontinuous on $S \times X$. As the sequence $\psi_{n}$ is bounded, we can choose a subsequence $\psi_{n^{\prime}}$ which is convergent to a continuous functional $\psi$.

By Lemma 2.2, we know that $\psi$ is the characteristic functional of a probability measure $\mu(t, \cdot), t \in S$. It is straightforward to show that the mapping $S \ni t \mapsto \mu(t, \cdot) \in \mathcal{M}(X)$ is an element of $W$ such that $\mu_{n^{\prime}} \mapsto \mu$ in the sense of $\mathcal{C}(S, \mathcal{M}(X))$.

Proof of Theorem 2.1. By Lemma 2.3 and assumption (A6), the proof is similar to the proof of the classical extreme value theorem. Uniqueness of $\bar{\mu}$ is an obvious consequence of (A7).

In what follows, we study the properties of the statistical solution constructed in [3]. In particular, we show that, assuming some kind of regularity of $F$, the demand $W \neq \emptyset$ is not necessary.

First, we recall some facts for completeness.

Set

$$
\mu_{n}^{0}=\mu_{0}\left(P_{n}^{-1}\left(\omega \cap X_{n}\right)\right), \quad \omega \in \mathcal{B}(X), n \in \mathbb{N} .
$$

DEFINITION 2.4. A statistical approximate solution of (1.1) is a family $\left\{\mu_{n}(t, \cdot)\right\}_{t \in[0, T]}$ of probability measures on $\mathcal{B}(X)$ such that

(1) $\operatorname{supp} \mu_{n}(t, \cdot) \subset X_{n}, t \in S$.

(2) For every $u^{*} \in X^{*}$ the characteristic functional of the measure $\mu_{n}(t, \cdot),[0, T] \ni t \mapsto \psi_{n}\left(t, u^{*}\right) \in \mathbb{C}$, is absolutely continuous.

(3) For almost all $t \in[0, T]$ and for every $u^{*} \in X^{*}$,

$$
\frac{\partial \psi_{n}\left(t, u^{*}\right)}{\partial t}=i \int_{X}\left\langle Q_{n} u^{*}, F(t, u)\right\rangle e^{i\left\langle u^{*}, u\right\rangle} \mu_{n}(t, d u) .
$$

(4) For every $u^{*} \in X^{*}$,

$$
\psi_{n}\left(0, u^{*}\right)=\int_{X} e^{i\left\langle u^{*}, u\right\rangle} \mu_{n}^{0}(d u) .
$$

For every $n \in \mathbb{N}$ and $t \in S$ we set

$$
F_{n}(t, \cdot)=\left.P_{n} \circ F(t, \cdot)\right|_{X_{n}} .
$$

The mapping $F_{n}(t, \cdot)$, written in the coordinates with respect to the basis of $X$, gives $n$ scalar functions

$$
F_{n}^{i}: S \times \mathbb{R}^{n} \ni\left(t, u_{1}, \ldots, u_{n}\right) \rightarrow F_{n}^{i}\left(t, u_{1}, \ldots, u_{n}\right) \in \mathbb{R}, \quad i=1, \ldots, n .
$$

It is assumed that 
(i) for all $n \in \mathbb{N}, t \in S$ and $i=1, \ldots, n$ the functions $F_{n}^{i}(t, \cdot)$ are of class $\mathcal{C}^{1}\left(\mathbb{R}^{n}\right)$

(ii) for all $n \in \mathbb{N}$ and $i=1, \ldots, n$ there exists $k_{n}^{i} \in L^{2}(S, \mathbb{R})$ such that for all $\left(u_{1}, \ldots, u_{n}\right) \in \mathbb{R}^{n}$ and for a.a. $t \in S$,

$$
\left|\frac{\partial F_{n}^{i}\left(t, u_{1}, \ldots, u_{n}\right)}{\partial u_{k}}\right| \leq k_{n}^{i}(t), \quad k=1, \ldots, n .
$$

THEOREM 2.5. Let $S_{n}$ be the translation along solution of the problem

$$
\begin{aligned}
u_{n}^{\prime}(t) & =F_{n}\left(t, u_{n}(t)\right), \\
u_{n}(0) & =P_{n} a .
\end{aligned}
$$

Let $\left\{\mu_{n}\right\}$ be the family of measures given by the formula

$$
\mu_{n}(t, \omega)=\mu_{0}\left(P_{n}^{-1}\left(S_{n}^{-1}(t, \omega)\right)\right), \quad \omega \in \mathcal{B}(X), t \in S .
$$

Then $\left\{\mu_{n}(t, \cdot)\right\}_{t \in[0, T]}$ is a statistical approximate solution of (1.1) with initial measure $\mu_{0}$. Moreover, there is $d>0$ such that for every $R \in \mathbb{R}$,

$$
\int_{E_{R}}\|u\| \mu_{n}(t, d u) \leq d \int_{E_{R / d-1}}(1+\|u\|) \mu_{0}(d u) .
$$

THEOREM 2.6. There exists a statistical solution of (1.1) with initial measure $\mu_{0}$.

Sketch of proof. Let $\psi_{n}: S \times X^{*} \rightarrow \mathbb{C}, n \in \mathbb{N}$, be the characteristic functional of $\mu_{n}$. In virtue of the Ascoli theorem there exists a subsequence $\left\{\psi_{n^{\prime}}\right\}$ such that

$$
\lim _{n^{\prime} \rightarrow \infty} \psi_{n^{\prime}}\left(t, u^{*}\right)=\psi\left(t, u^{*}\right), \quad\left(t, u^{*}\right) \in S \times X^{*},
$$

where $\psi: S \times X^{*} \rightarrow \mathbb{C}$ is a continuous functional. Without loss of generality we may assume that $\psi_{n^{\prime}}$ coincides with $\psi_{n}$.

By Lemma 2.2 we can show that there exists a family of $\{\mu(t, \cdot)\}$ on $\mathcal{B}(X)$ such that

$$
\begin{gathered}
\int_{X}\|u\| \mu(t, d u)<\infty, \quad t \in S, \\
\psi\left(t, u^{*}\right)=\int_{X} \exp \left(i\left\langle u^{*}, u\right\rangle\right) \mu(t, d u), \quad u^{*} \in X^{*} .
\end{gathered}
$$

This family is a statistical solution of (1.1) with initial measure $\mu_{0}$. For more details we refer the reader to [3].

THEOREM 2.7. The statistical solution constructed above satisfies conditions (A4), (A5) (with d taken from (2.7)).

Proof. It can be shown that for $t \in S, k \in \mathbb{N}, R \in \mathbb{R}$,

$$
\liminf _{n \rightarrow \infty} \int_{E_{R}(k)}\left\|P_{k} u\right\| \mu_{n}(t, d u) \geq \int_{E_{R}(k)}\left\|P_{k} u\right\| \mu(t, d u),
$$

where $E_{R}(k)=\left\{u \in X:\left\|P_{k} u\right\|>R\right\}$. 
As a consequence of (2.7) and (2.8), we have

$$
\int_{E_{R}(k)}\left\|P_{k} u\right\| \mu(t, d u) \leq d \int_{E_{R / d-1}}(1+\|u\|) \mu_{0}(d u) .
$$

This inequality, together with the observation that

$$
\lim _{k \rightarrow \infty} \int_{E_{R}(k)}\left\|P_{k} u\right\| \mu(t, d u)=\int_{E_{R}}\|u\| \mu(t, d u),
$$

finishes the proof that condition (A4) is satisfied.

We have

$$
J=\lim _{k \rightarrow \infty} J_{k}
$$

where $J_{k}: S \ni t \mapsto \int_{X} f\left(P_{k} u\right) \mu(t, d u), k \in \mathbb{N}$, are continuous functions. Hence $J$ is measurable and, by (A4), condition (A5) is easily verified.

An important example of a functional $\mathcal{F}$ is related to the average value of a measure. Recall that if $\mu$ is a probability measure on $\mathcal{B}(X)$ with

$$
\int_{X}\|u\| \mu(d u)<\infty
$$

then there exists $E(\mu) \in X$ such that

$$
\left\langle u^{*}, E(\mu)\right\rangle=\int_{X}\left\langle u^{*}, u\right\rangle \mu(d u), \quad u^{*} \in X^{*} .
$$

$E(\mu)$ is called the average value of $\mu$.

For $\{\mu(t, \cdot)\}_{t \in S} \in W$ we set

$$
\mathcal{F}\left(\{\mu(t, \cdot)\}_{t \in S}\right)=\int_{0}^{T}\|E(\mu(t, \cdot))\|^{2} d t .
$$

It is easily checked that the functional $\mathcal{F}$ is lower semicontinuous and strongly convex on $W$.

\section{References}

[1] C. Foiaş, Statistical study of Navier-Stokes equations, I, Rend. Sem. Mat. Univ. Padova 48 (1972), 219-348.

[2] - Statistical study of Navier-Stokes equations, II, ibid. 49 (1973), 282-313.

[3] L. Sławik, Evolution of measure governed by differential equations, Int. J. Differ. Equ. Appl. 1A (2000), 195-204.

[4] M. I. Višik et A. V. Foursikov, L'équation de Hopf, les solutions statistiques, les moments correspondants aux systèmes des équations paraboliques quasilinéaires, J. Math. Pures Appl. 56 (1977), 85-122.

Institute of Mathematics

Cracow University of Technology

31-155 Kraków, Poland

E-mail: lslawik@usk.pk.edu.pl 